\title{
Elaboração de cupcake adicionado de farinha de fibra de caju: caracterização físico-química e sensorial
}

Elaine Carvalho de Morais elaine carvalho.2@hotmail.com Departamento de Ensino, Pesquisa e Extensão, Instituto Federal de Mato Grosso, Campus

Cuiabá, Cuiabá, Mato Grosso, Brasil.

Samira Gabrielle Oliveira Patias $\frac{\text { samiragabrielle@hotmail.com }}{\text { Departamento de Alimentos e Nutrição, }}$ Universidade Federal do Mato Grosso, Cuiabá, Mato Grosso, Brasil.

\section{Erika Silva Costa}

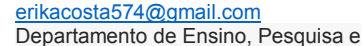
Extensão, Instituto Federal de Mato Grosso, Campus

Cuiabá, Cuiabá, Mato Grosso, Brasil.

Dayane de Oliveira Sandri dayanesandri@gmail.com Departamento de Ensino, Pesquisa e Extensão, Instituto Federal de Mato Grosso, Campus

Cuiabá, Cuiabá, Mato Grosso, Brasil.

Nágela Farias Magave Picanço

nagelap@terra.com.br Tecnologia de Alimentos, Instituto Federal de Mato Grosso, Campus Cuiabá, Cuiabá, Mato Grosso, Brasil.

\section{Rozilaine Aparecida Pelegrine} Gomes de Faria

rozilaine.faria@blv.ifmt.edu.br

Programa de Pós-Graduação em Ciência e Tecnologia de Alimentos, Instituto Federal de Mato Grosso, Campus Cuiabá, Cuiabá, Mato Grosso, Brasil.

\begin{abstract}
RESUMO
O objetivo neste estudo foi avaliar a composição físico - química e aceitação sensorial de cupcake elaborado com substituição parcial de farinha de trigo pela farinha da fibra de caju (FFC). Foram elaboradas três formulações de cupcake adicionados de FFC, uma padrão (F1) sem FFC e as demais com $5 \%$ de FFC (F2) e 12\% FFC (F3). Os cupcakes foram elaborados conforme receita padrão. Foi avaliada a composição centesimal das formulações, conforme metodologia da AOAC (2012). A análise sensorial foi realizada com 70 provadores não treinados. Os atributos avaliados foram aparência, aroma, sabor, textura e aceitação global, mediante escala hedônica estruturada de nove pontos. As médias obtidas de cada análise foram submetidas a análise de variância e quando significativas comparadas pelo teste de Tukey a 5\%. Não houve diferença significativa entre os atributos da análise sensorial, exceto para a aparência do tratamento sem farinha de fibra de caju que foi atribuída maior média entre os provadores. Todas as formulações tiveram um alto nível de aceitação maior que $82 \%$. Os cupcakes elaborados com FFC apresentaram maior quantidade de fibras quando comparada com F1. A adição de farinha de fibra de caju mostrou-se uma boa alternativa para o aproveitamento desse subproduto, assim reforça o desenvolvimento de produtos alimentícios com o resíduo do caju, reduzindo perdas e agregando valor e nutrientes a esses produtos.
\end{abstract}

PALAVRAS-CHAVE: Pedúnculo do caju; aproveitamento de alimento; segurança alimentar; resíduo de caju; valor nutricional; aceitação sensorial. 


\section{INTRODUÇÃO}

De acordo com relatórios publicados pela Organização das Nações Unidas para a Alimentação e Agricultura (FAO) em 2013, anualmente cerca de 1,3 bilhões de toneladas de alimentos é desperdiçada no mundo. $O$ desperdício ocorre devido a vários fatores como nas etapas da produção, da manipulação pós-colheita e da armazenagem dos produtos, que é responsável por $54 \%$ e se concentra nos países em desenvolvimento. Já o processamento, a distribuição e o consumo são responsáveis pelos $46 \%$ restantes desperdiçados, sendo mais constatados nas regiões mais industrializadas ou de renda média mais elevada (FAO, 2013; PEIXOTO; PINTO, 2016).

No Brasil o desperdício é um caso peculiar, aproximadamente um terço dos alimentos produzidos é desperdiçado, assim a redução do desperdício pode ser uma forte aliada no combate a insegurança alimentar e o aproveitamento integral dos alimentos pode contribuir para a melhoria das dietas oferecidas para os consumidores e melhorar os hábitos alimentares da população (FAO, 2017; PORPINO, 2016).

Dados da Associação Brasileira das Indústrias de Biscoitos, Massas alimentícias e Pães \& Bolos Industrializados (ABIMAPI) revelaram que em 2014 o volume total de vendas de bolo atingiu cerca de 40,6 mil toneladas e o consumo per capita ficou em $0,20 \mathrm{~kg} /$ ano. Esse produto vem ganhando espaço chegando a $50,7 \%$ dos lares brasileiros, o que leva a reforçar o empenho das indústrias nesse segmento. O Brasil ocupa a sexta colocação global no ranking de vendas de bolos industrializados (ABIMAPI, 2016). A categoria de pães e bolos industrializados representa os produtos que serão mais consumidos em 2020, conforme Brazil Food Trend 2020.

Embora sejam apontados como produtos de alto valor calórico, os cupcakes podem ser produzidos com adição de fibra de caju, fornecendo os benefícios inerentes as fibras alimentares, evitando assim o desperdício (PINHO, 2009). E a incorporação de fibras na alimentação da população aumenta a saciedade e colabora com a manutenção do peso e a oferta de alimento com melhor qualidade nutricional estimulando a prática de hábitos saudáveis (FRANZEN et al., 2014).

O caju apresenta um elevado valor econômico, devido à qualidade e valor nutricional de sua castanha que possui vitaminas e ácidos graxos monoinsturados, além da riqueza de vitamina $\mathrm{C}$ e fibras de seu pedúnculo, que corresponde à parte polposa e comestível (pseudofruto) (EMBRAPA, 2003). Dependendo da variedade do fruto, pode-se obter boa qualidade na produção de fibras. Lima, García e Lima (2004) obtiveram bom teor de fibra dietética total para a polpa do pedúnculo do caju, 61\%, e Rufino et al. (2010) concluíram que o bagaço de caju apresenta elevada capacidade antioxidante e que parte significativa dos polifenóis está associada com a fibra dietética, o que justifica melhor aproveitamento das fibras presentes na polpa.

O aproveitamento do pseudofruto somente na forma de suco produz resíduo geralmente descartado. E esse desperdício resulta no não aproveitamento dessas fibras descartadas e presentes na polpa, aumentando a quantidade de resíduos não aproveitados. O aproveitamento do fruto na sua totalidade com outras possibilidades de incorporação dos nutrientes na dieta poderia corroborar com aumento da ingestão de fibras e melhoria de renda das comunidades rurais. $\mathrm{E}$ 
também possibilitar a diversidade de cardápio da região nos formatos de cupcakes agregando valor nutricional através da incorporação das fibras (UCHOA et al., 2008).

A nova tendência de mercado para alimentação do tipo smallfoods corrobora com a diversidade na oferta de alimentos. Agregar frutas com pratos tradicionais apresentados de formas variadas, entre eles cupcake melhora a qualidade nutricional e facilita a aceitação por públicos mais jovens (MENEGUEL; SILVA, 2012).

Vários pesquisadores nos últimos anos vêm estudando o aproveitamento de resíduos agroindustriais como fonte de fibra como casca de abacaxi (MARTINS et al., 2012), semente de abóbora (BITENCOURT et al.,2014), bagaço de mandioca (SILVA et al., 2016) e bagaço de caju adicionado a hambúrgueres (GALVÃO, 2006; BARROS et al., 2012), doces (SUCUPIRA et al., 2014; COSTA et al., 2016), barra de cereal (OLIVEIRA et al., 2013), além disto a farinha deste bagaço empregada na confecção de biscoito ( SABINO et al., 2017) e pão (MARQUES et al., 2008) foi bem aceita pelos consumidores. Assim a utilização de resíduos pode contribuir de forma significativa para alimentação saudável e para novos processos tecnológicos.

Nesse contexto, o objetivo deste trabalho foi avaliar a composição físicoquímica e aceitação sensorial de cupcake elaborado em substituição parcial de farinha de trigo pela farinha da fibra de caju.

\section{MATERIAIS E MÉTODOS}

\section{MATÉRIA-PRIMA}

Os cajus foram adquiridos em pontos de venda do município de Cuiabá - MT e transportados em caixas térmicas até as instalações do Laboratório de Processamento de Alimentos do Instituto Federal de Mato Grosso. Foram eliminados os pedúnculos verdes, machucados e excessivamente maduros, em seguida foram sanitizados durantes $30 \mathrm{~min}$ em água contendo $150 \mathrm{ppm}$ de cloro livre e enxaguados em água corrente (LIMA, GARCÍA e LIMA, 2004). Depois foi extraído manualmente o suco da polpa para obtenção da fibra úmida. Posteriormente para obtenção da farinha alimentícia, as fibras foram lavadas sucessivamente com água e posteriormente desidratadas em estufa com circulação de ar forçada a $60{ }^{\circ} \mathrm{C}$ durante 6 horas. Após secagem as fibras foram trituradas em moinho de bolas modelo MA 350 da marca Marconi ${ }^{\circledR}$, peneiradas em peneira de 40 mesh e acondicionadas em frascos fechados armazenados a temperatura ambiente (LIMA, GARCÍA e LIMA, 2004; UCHOA et al., 2008).

\section{DESENVOLVIMENTO DOS CUPCAKES}

A partir da farinha de fibra de caju (FFC) foi desenvolvida três formulações de cupcake em diferentes proporções (F1 = 0\%; F2 = 5\%; F3 = 12\%) em substituição da farinha de trigo. As formulações foram preparadas individualmente, com as proporções dos ingredientes de acordo com a Tabela 1, definidos através de testes preliminares. 
Para o preparo dos cupcakes em uma batedeira comum, foram batidos a margarina e o açúcar refinado até a obtenção de um creme esbranquiçado, em seguida adicionaram-se os ovos um a um com a batedeira ligada. Logo após acrescentaram-se os demais ingredientes obtendo uma massa homogênea. 0 fermento químico foi misturado manualmente. Em seguida a massa foi distribuída em forminhas de papel posicionadas sobre as de alumínio e levadas ao forno préaquecido a 200 으 por 40 minutos.

Tabela 1. Ingredientes das formulações de cupcake adicionados de FFC.

\begin{tabular}{lccc}
\hline \multirow{2}{*}{ Ingredientes (\%)* } & \multicolumn{3}{c}{ Formulações } \\
\cline { 2 - 4 } & $\mathrm{F} 1$ & $\mathrm{~F} 2$ & $\mathrm{F3}$ \\
\hline Farinha de trigo & 100 & 95 & 88 \\
Farinha de fibra de caju - FFC & 0 & 5 & 12 \\
Margarina & 62,5 & 62,5 & 62,5 \\
Açúcar refinado & 100 & 100 & 100 \\
Ovos & 15 & 15 & 15 \\
Leite integral & 62,5 & 62,5 & 62,5 \\
Fermento químico & 5 & 5 & 5 \\
\hline
\end{tabular}

NOTA: * Valores dos ingredientes referentes a $100 \%$ do peso total da farinha de trigo. F1: padrão 0\% de FFC; F2: 5\% de FFC e F3: $12 \%$ de FFC.

\section{ANÁLISE SENSORIAL}

A análise sensorial foi aprovada pelo Comitê de Ética em Pesquisa Envolvendo Seres Humanos da Secretaria de Estado de Saúde de Mato Grosso, SES/MT ( $n$ o do Parecer 1.333.947). As formulações foram submetidas à análise sensorial quanto aos atributos aparência, aroma, sabor, textura e aceitação global, mediante escala hedônica estruturada de nove pontos ( $1=$ desgostei muitíssimo, $9=$ gostei muitíssimo). Foi aplicado também o teste de intenção de compra avaliada por escala hedônica estruturada de cinco pontos ( 1 = certamente não compraria, $5=$ certamente compraria).

O teste foi conduzido em cabines individuais com iluminação de cor branca, com 70 provadores não treinados abordados aleatoriamente, entre professores, alunos e funcionários do Instituto Federal de Mato Grosso, campus Cuiabá - Bela Vista, de ambos os sexos, com idade entre 18 e 60 anos.

Cada julgador recebeu amostras de aproximadamente $10 \mathrm{~g}$ de cada formulação, servidas e codificadas com números de três dígitos de forma balanceada e casualizada, acompanhados de um copo de água, conforme método desenvolvido por Minim (2006).

O cálculo do Índice de Aceitabilidade (IA) de cada formulação foi conforme a expressão IA $(\%)=(A \times 100) / B$, onde $A$ é a nota média obtida para o produto e $B$ a nota máxima dada ao produto. 


\section{ANÁLISE DA COMPOSIÇÃO QUÍMICA}

Foi realizada determinação da composição centesimal de cada formulação e da FFC, em triplicata, segundo os procedimentos recomendados pela Association of Official Analytical Chemistry (AOAC, 2012) e Instituto Adolfo Lutz (IAL, 2008).

Os teores de umidade (estufa $105^{\circ} \mathrm{C} / 24$ horas) e cinzas (mufla $550{ }^{\circ} \mathrm{C} / 4$ horas) foram determinados através do método gravimétrico, método no 920.151 e no 920.153 respectivamente. A determinação de lipídeos foi realizada pelo método de Soxhet (método 991.36), o teor de proteínas foi determinado pelo método Kjedahl (método 928.08), o qual é baseado na determinação de nitrogênio, usando-se o fator 6,25 para a conversão do nitrogênio total em proteína total. Os carboidratos foram calculados de acordo com as análises de açúcar redutor, não redutores e totais utilizando o método de Fehling (IAL, 2008). A fibra bruta foi determinada por método gravimétrico no qual as amostras foram submetidas à digestão ácida, com solução de ácido sulfúrico 1,25\%, seguida por digestão alcalina com hidróxido de sódio. Após as digestões, o resíduo foi submetido a aquecimento a $105-110^{\circ} \mathrm{C}$ e pesado até massa constante. Posteriormente, esse foi calcinado a $550^{\circ} \mathrm{C}$ até massa constante. (IAL, 2008).

O valor energético total (VET) foi calculado de acordo com o valor energético dos macronutrientes considerando os fatores de conversão de Atawer de $4 \mathrm{kcal} / \mathrm{g}$ de proteína e carboidrato e $9 \mathrm{kcal} / \mathrm{g}$ de lipídio.

\section{ANÁLISE ESTATÍ́STICA}

Os dados da análise sensorial e físico-química foram avaliados através de análise de variância (ANOVA) e teste de Tukey para comparação das médias com nível de significância a 5\%, utilizando o programa ASSISTAT versão beta 7.0.

\section{RESULTADO E DISCUSSÃO}

Os resultados da análise sensorial das formulações de cupcake estão apresentados na Tabela 2.

Tabela 2. Médias das notas da análise sensorial dos atributos avaliados, para cada formulação de cupcake elaborada com FFC.

\begin{tabular}{lccc}
\hline \multirow{2}{*}{ Atributos } & \multicolumn{3}{c}{ Formulações ** } \\
\cline { 2 - 4 } & $\mathrm{F} 1$ & $\mathrm{~F} 2$ & $\mathrm{~F} 3$ \\
\hline Aparência & $8,19^{\mathrm{a}}$ & $7,60^{\mathrm{b}}$ & $7,39^{\mathrm{b}}$ \\
Aroma & $7,94^{\mathrm{a}}$ & $7,70^{\mathrm{a}}$ & $7,71^{\mathrm{a}}$ \\
Sabor & $7,90^{\mathrm{a}}$ & $7,53^{\mathrm{a}}$ & $7,60^{\mathrm{a}}$ \\
Textura & $8,11^{\mathrm{a}}$ & $7,70^{\mathrm{a}}$ & $7,90^{\mathrm{a}}$ \\
Aceitação Global & $8,00^{\mathrm{a}}$ & $7,66^{\mathrm{a}}$ & $7,69^{\mathrm{a}}$ \\
\hline
\end{tabular}

NOTA: Letras diferentes na linha indicam diferença significativa pelo Teste de Tukey $(p<0,05)$; ** F1: padrão; F2: $5 \%$ e F3: $12 \%$ de FFC. significativa em relação à formulação padrão, porém as duas não se diferenciaram. 
Quanto maior a concentração da farinha menor a nota para o quesito aparência. Isso indica que a coloração escura atribuída pela massa de FFC não se mostrou atrativa para os provadores. Não houve diferença significativa $(p>0,05)$ entre os demais atributos, para as três formulações e apresentam escores satisfatórios entre 7 (gostei moderadamente) e 8 (gostei muito).

Alves et al. (2011) avaliaram a aceitação de produtos de panificação utilizando a farinha de caju em substituição da farinha de trigo e obteve nota semelhante a deste estudo, quando comparada com o atributo aparência, onde obteve valor de 6,95 para formulação com $20 \%$ de farinha de caju.

Já Barros et al. (2012) elaboraram hambúrguer com fibra de caju e obtiveram melhor aceitação na formulação com $30 \%$ que apresentou bom valor nutritivo, com maior conteúdo de fibras e menor teor de gordura quando comparado ao hambúrguer tradicional.

De modo geral a formulação F1 recebeu as maiores notas dos avaliadores, apresentando notas em média de 7 (gostei moderadamente) e 8 (gostei muito) em todos os quesitos avaliados. Mas isso não implica rejeição das demais amostras, pois as mesmas também apresentam um bom escore, mostrando que mesmo os cupcakes elaborados com $12 \%$ de farinha de fibra de caju foram bem aceitos pelos provadores.

Em relação ao índice de aceitabilidade (IA) apontado na Figura 1, observa-se que todas as formulações apresentaram IA acima de 70\%, considerando assim o produto com boa aceitação sensorial, de acordo com Dutcoski (2011).

Bitencourt et al. (2014) realizaram análise sensorial de bolo formulado com farinha de semente abóbora e demonstraram IA superior a 70\% com exceção da formulação com $30 \%$ para o atributo aparência e cor.

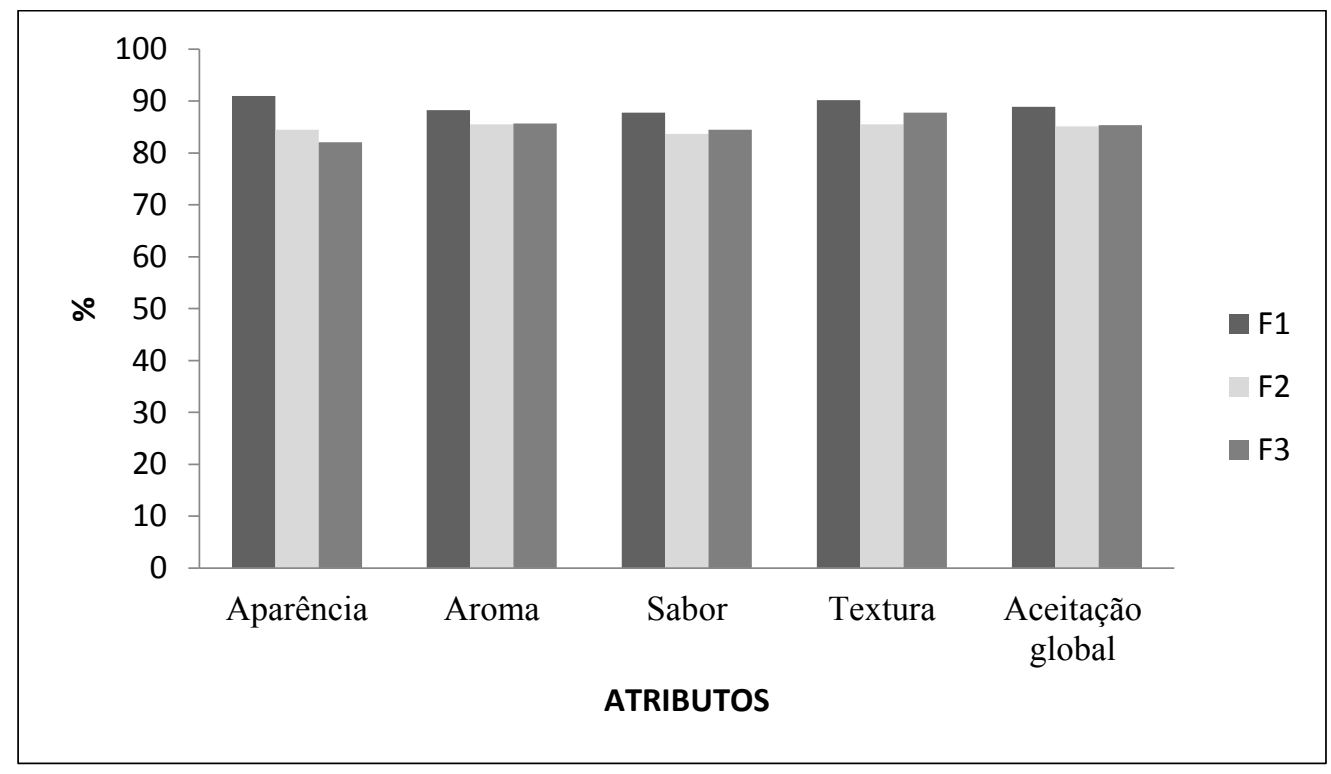

Figura 1 - Índice de aceitabilidade das formulações de cupcake padrão (F1) e adicionadas de $5 \%$ (F2) e $12 \%$ (F3) de FFC, em relação aos atributos avaliados. 
De modo geral, a formulação F1 apresentou maiores médias para todos os atributos, porém a F3 $(84,44 \%)$ teve maior índice de aceitação no atributo sabor quando comparada a F2 $(83,63)$. Mostrando que adição de até $12 \%$ de FFC é aceitável entre os provadores. O costume da população por bolos preparados com farinha de trigo dificulta a aceitação quando adicionado outros tipos de farinha como relata Vieira et al. (2010).

Em relação à intenção de compra $69 \%$ dos julgadores certamente comprariam o produto, sendo as formulação F1 e F2 as mais escolhidas apresentando 35 e $40 \%$ respectivamente. Isso demonstra que os provadores "certamente compraria" o cupcake com adição de $5 \%$ de FFC podendo ser um produto viável para comercialização.

\section{ANÁLISE DA COMPOSIÇÃO CENTESIMAL}

$\mathrm{Na}$ Tabela 3 estão apresentados os resultados da composição centesimal e valor energético dos cupcakes elaborados.

Tabela 3. Valores médios \pm desvio padrão dos parâmetros físico-químicos de cupcake elaborados sem farinha de fibra de caju (F1) e adicionados de $5 \%$ (F2) e $12 \%$ (F3) de FFC.

\begin{tabular}{cccc}
\hline Parâmetros & $F 1$ & $F 2$ & $F 3$ \\
\hline Umidade & $25,19^{\mathrm{a}} \pm 0,15$ & $23,24^{\mathrm{c}} \pm 0,07$ & $24,80^{\mathrm{b}} \pm 0,07$ \\
Cinzas & $1,05^{\mathrm{a}} \pm 0,02$ & $1,16^{\mathrm{a}} \pm 0,03$ & $1,12^{\mathrm{a}} \pm 0,07$ \\
Proteínas & $6,34^{\mathrm{a}} \pm 0,11$ & $6,19^{\mathrm{a}} \pm 0,10$ & $6,23^{\mathrm{a}} \pm 0,07$ \\
Lipídeos & $13,44^{\mathrm{a}} \pm 0,21$ & $13,44^{\mathrm{a}} \pm 0,28$ & $14,34^{\mathrm{a}} \pm 0,08$ \\
Açúcar redutor & $2,66^{\mathrm{c}} \pm 0,01$ & $4,66^{\mathrm{b}} \pm 0,08$ & $6,54^{\mathrm{a}} \pm 0,01$ \\
Açúcares totais & $9,39^{\mathrm{a}} \pm 0,31$ & $9,14^{\mathrm{a}} \pm 0,42$ & $7,48^{\mathrm{b}} \pm 0,38$ \\
Açúcar não redutor em sacarose & $6,86^{\mathrm{a}} \pm 0,31$ & $4,71^{\mathrm{b}} \pm 0,44$ & $1,26^{\mathrm{c}} \pm 0,06$ \\
Fibras & $0,49^{\mathrm{c}} \pm 0,01$ & $0,96^{\mathrm{b}} \pm 0,04$ & $2,78^{\mathrm{a}} \pm 0,08$ \\
Valor calórico (Kcal) & $361,82^{\mathrm{a}} \pm 0,78$ & $369,80^{\mathrm{b}} \pm 0,79$ & $367,88^{\mathrm{b}} \pm 0,72$
\end{tabular}

NOTA: Letras iguais na mesma linha não diferem estatisticamente pelo teste de Tukey a 5\% de probabilidade.

A adição de farinha de caju a formulação apresentou diferença significativa $(p<0,05)$ para umidade, variando de $25,19 \%$ para o padrão e $24,80 \%$ para formulação com $12 \%$ de FFC. Alves et. al (2011), encontrou valores de 10,83\%; 3,18\%; 4,8\% para umidade, cinzas e proteína para farinha de caju.

Quando avaliado estatisticamente os resultados do parâmetro de cinzas, proteínas e lipídeos não houve diferença significativa $(p>0,05)$. Isso ocorre devido a farinha de caju (FC) apresentar resultados baixo para esses atributos, devem-se também aos demais ingredientes que foram acrescentados em proporções iguais para cada formulação.

O açúcar redutor e não redutor teve diferença significativa $(p<0,05)$ entre as formulações. Lima, García e Lima (2004) relataram que a fibra do pedúnculo do caju apresenta boas quantidades de açúcares redutores $34,60 \%$, isso pode explicar 
porque a formulação F3 apresentou maior teor de açúcar redutor quando comparada com a formulação $\mathrm{F} 1$ devido à presença desses glicídios simples. $\mathrm{O}$ pseudofruto do caju possui monossacarídeos próprios e possibilita a substituição parcial da sacarose diminuindo custos na elaboração do produto, pois a presença desses monossacarídeos permite a percepção sensorial de sabor mais adocicado.

A quantidade de fibra bruta encontrada neste estudo é um dos pontos principais, as formulações diferenciaram estatisticamente $(p<0,05)$ e houve um aumento $567 \%$ em relação a formulação padrão quando comparado com cupcake acrescido de $12 \%$ de FFC. Isso se deve ao alto teor de fibra presente na FC, como verificado por Matias et al. (2005), que encontrou valor de 41,53 g/100g de fibra em bagaço desidratado de caju.

A FFC deste estudo apresentou valor de 39,9 $\pm 0,13(\mathrm{~g} / 100 \mathrm{~g})$ de fibra bruta, um teor bem elevado e que pode ser adicionada a dietas melhorando a qualidade nutricional, sendo uma opção de aplicação em produtos preparados comumente com farinhas de cereais. Por ter baixo custo e alto valor nutritivo os resíduos agroindustriais, pouco explorados, podem ser uma alternativa viável para enriquecer diversos alimentos com nutrientes como fibras, proteínas, flavonoides e etc. (MIRANDA et al., 2013).

A ingestão diária de fibras recomendada segundo a Dietary Reference Intakes (DRI) é de $25 \mathrm{~g}$ para mulheres e $38 \mathrm{~g}$ para homens, ambos com idades entre $19 \mathrm{e}$ 50 anos. Dessa forma produtos enriquecidos com FFC como o deste estudo pode ser um aliado na inserção de fibras na alimentação da população, melhorando a qualidade dos alimentos ofertados e por outro lado diminuindo o desperdício que ocorre em todos os elos da cadeia produtiva.

De acordo com a Portaria $n^{\circ} 27$ do Ministério da Saúde, referente à informação nutricional, o produto com no mínimo $3 \%$ de fibras pode ser considerado como fonte de fibras e ao ultrapassar o mínimo de $6 \%$ será considerado com alto teor de fibras (BRASIL, 1998). O produto analisado chegou próximo ao valor para ser considerado como fonte de fibras.

\section{CONCLUSÃO}

Os cupcakes apresentaram um alto nível de aceitação, maior que $82 \%$. A elaboração dos produtos permitiu comprovar que um nível de adição de até $12 \%$ de FFC em cupcakes foi bem aceito pelos provadores, obtendo-se aceitação sensorial semelhante ao produto padrão. A adição de $12 \%$ de FFC proporcionou aumento no aporte de fibras, melhorando o perfil nutricional do produto. Assim sendo, a farinha da fibra de caju pode ser considerada como um potencial ingrediente para adição em bolos e similares, podendo ser oferecida aos consumidores com altas expectativas de aceitação no mercado, além de ser alternativa para o aproveitamento desse subproduto com redução de perdas e agregação de valor.

\section{AGRADECIMENTOS}

Os autores agradecem ao fomento recebido pelo IFMT, a UNIC - Universidade de Cuiabá pelo auxilio nas análises, a CAPES pela bolsa concedida ao autor, a bolsa 
Pibit/CNPq concedida a segunda co-autora, ao CNPq pela bolsa de estudos na modalidade Desenvolvimento Tecnológico e Industrial concedida a terceira coautora. 


\title{
Elaboration of added capucake of cashew fiber flour: physico-chemical and sensory characterization.
}

\begin{abstract}
The objective of this study was to evaluate the physical - chemical composition and sensorial acceptance of cupcake elaborated in partial replacement of wheat flour with cashew fiber flour (FFC). Three cupcake formulations were added with FFC, one standard (F1) without FFC and the other with 5\% FFC (F2) and 12\% FFC (F3). The cupcakes were made according to standard recipe. The centesimal composition of the formulations was evaluated, according to AOAC methodology (2012). Sensory analysis was performed with 70 untrained testers. The evaluated attributes were appearance, aroma, flavor, texture and global acceptance, using a structured hedonic scale of nine points. The mean values were subjected to analysis of variance and were submitted to the Tukey test at $5 \%$ significance level. There was no significant difference between the attributes of the sensory analysis, except for the appearance of the treatment without cashew fiber flour that was assigned the highest average among the tasters. All formulations had a high level of acceptance greater than $80 \%$. Cupcakes made with FFC presented higher amounts of fiber when compared to F1. The addition of cashew fiber flour proved to be a good alternative for the utilization of this by-product, thus reinforcing the development of food products with the cashew residue, reducing losses and adding value and nutrients to these products.
\end{abstract}

KEYWORDS: Cashew peduncle; food utilization; food safety; cashew residue; nutritional value; sensorial acceptance. 


\section{REFERÊNCIAS}

A.O.A.C. Association of Official Analytical Chemists. Official Methods of Analysis of the Association of Analytical Chemistry.13 ed. Washington, 2012.

ABIMAPI - ASSOCIAÇÃO BRASILEIRA DAS INDÚSTRIAS DE BISCOITOS, MASSAS ALIMENTÍCIAS, PÃES \& BOLOS INDUSTRIALIZADOS. Estatísticas: pães \& bolos. Disponível em:< http://www.abimapi.com.br/estatistica-paes-bolos.php $>$. Acesso em: 29 de nov. de 2016.

ALVES, F. M. S.; MACHADO, A. V.; QUEIROGA, K. H. Alimentos produzidos a partir de farinha de caju, obtida por secagem. Revista Verde, v. 6, n. 3, p. 131-138, 2011.

BARROS, N. V. A.; COSTA, N. Q.; PORTO, R. G. C. L.; MORGANO, M. A.; ARAÚJO, M. A. M.; MOREIRA-ARAÚJO, R. S. dos R. Elaboração de hambúrguer enriquecido com fibras de caju (Anacardium occidentale L.). Boletim CEPPA, Curitiba, v. 30, n. 2, p. 315-325, jul./dez. 2012.

BITENCOURT, C.; DUTRA, F. L. G.; PINTO, A. Z.; HELBIG, E.; BORGES, L. R. Elaboração de bolos enriquecidos com semente de abóbora: avaliação química, física e sensorial. Botetim CEPPA, v. 32, n. 1, p. 19-32, 2014.

BRASIL. Ministério da Saúde (BR). Portaria no 27 de 13 de janeiro de 1998. Regulamento técnico referente à informação nutricional complementar. Diário Oficial da República Federativa do Brasil. 11 jul 1998; Seção 1.

BRAZIL FOOD TRENDS 2020. FIESP/DEAGRO; Instituto de Tecnologia de Alimentos/ITAL. São Paulo: Gráfica Ideal, 2010. Disponível em: <http://www.brasilfoodtrends.com.br/Brasil_Food_Trends/index.html>. Acesso em: 29 nov. 2016.

COSTA, J. A.; RODRIGUES, A. M. D.; SANTOS, J. T. O.; ALENCAR, A. C.; PIRES, R. M. C.; NOBREGA, M. M. G. P.; MURATORI; M. C. S. Avaliação microbiológica e sensorial de doce em pasta elaborado com soro de leite e pedúnculo do caju. Acta Veterinaria Brasilica, v. 10, n. 1, p. 9-15, 2016.

DUTCOSKY, S. D. Análise sensorial de alimentos. 3. ed. Curitiba: Champagnat, 2011. 426p.

EMBRAPA AGROINDUSTRIA TROPICAL, SERVIÇO BRASILEIRO DE APOIO ÀS MICRO E PEQUENAS EMPRESAS. Iniciando um pequeno grande negócio agroindustrial: castanha de caju. Brasília: Embrapa Informação Tecnológica, 2003. 131 p. 
FAO. 2017. Panorama de la seguridad alimentaria y nutricional 2016: Sistemas alimentarios sostenibles para poner fin al hambre y la malnutrición. Santiago, 2017. Disponível em:< http://www.fao.org/3/a-i6747s.pdf>. Acesso em: 10 de jan. 2017.

FAO. 2013. Food wastage footprint, Impacts on natural resources. Summary Report. FAO, Roma. Disponível em:

<http://www.fao.org/docrep/018/i3347e/i3347e.pdf>. Acesso em: 25 nov. 2016.

FRANZEN, J. M.; NUNES, T. R. G.; ZANCANARO, T. F. V. Elaboração e análise sensorial de cupcakes preparados a partir da farinha de semente de abóbora (Curcubita maxima) em crianças de 7 anos de idade. RIES, v. 3, n. Especial, p. 7-12, 2014.

GALVÃO, A. M. P. Aproveitamento da fibra de caju (Anacardium occidentale, L.) na formulação de um produto tipo hambúrguer. 75 f. Dissertação (Mestrado em Tecnologia de Alimentos) - Universidade Federal do Ceará, Fortaleza, 2006.

INSTITUTO ADOLFO LUTZ. Métodos físico-químicos para análise de alimentos. Ed. IV, 2008, 1018p.

LIMA, A. C.; GARCÍA, N. H. P.; LIMA, J. R. Obtenção e caracterização dos principais produtos do caju. Boletim CEPPA, v. 22, n. 1, p. 133-144, 2004.

MARQUES, L. F.; COSTA, T. L.; MOURA, R. L.; COSTA, A. M. N. M.; CHAVES, C. G.; FREITAS, R. M.; ROCHA, E. M. F. F.; MOURA, L. B. Produção e aceitação sensorial de pão tipo hambúrguer fabricado com adição de $10 \%$ de farinha do bagaço de caju. In: III Jornada Nacional da Agroindústria. Bananeiras, Paraíba: Resumos, s.n., 2008.

MARTINS, J. G. P. JÚNIOR, M. D. da M.; ALMEIDA, M. A.; SANTOS, T.; SPOTO, M. H. F. Avaliação sensorial de bolo com resíduo de casca de abacaxi para suplementação do teor de fibras. Revista Brasileira de Produtos Agroindustriais, v. 1, n. 3, p. 281287, 2012

MATIAS, M. F. O.; OLIVEIRA, E. L.; GERTRUDES, E.; MAGALHÃES, M. M. A. Use of fibres obtained from cashew (Anacardium ocidentale, $\mathrm{L}$ ) and guava (Psidium guayava) fruits for enrichment of food products. Brazilian Archives of Biology and Technology, v. 48, n. special, p. 143-150, 2005.

MENEGUEL, C. R. A.; SILVA, A. M. S. Tendências gastronômicas no mercado de eventos e sua metodologia integradora. In: Simpósio do Ensino Médio e Técnico: 
Práticas Integradoras e Gestão de Currículo. Atibaia, São Paulo: Anais, p. 48-53, 2012.

MINIM, V. P. R. Analise Sensorial: Estudos com Consumidores. 2. ed. Editora: Fundação Arthur Bernardes - Editora UFV, 2006.

MIRANDA, A. A.; CAIXETA, A. C. A.; FLÁVIO, E. F.; PINHO, L. Desenvolvimento e análise de bolos enriquecidos com farinha da casca do maracujá (Passiflora Edulis) como fonte de fibras. Alimentos e Nutrição, Brazilian Journal Food and Nutrition, Araraquara, v. 24, n. 2, p. 225-232, abr./jun. 2013.

OLIVEIRA, C. F. P.; MALTA, H. L.; JESUS, M. A. C. L.; CRUZ, R. S.; CARDOSO, F. S. N. Desenvolvimento, avaliação sensorial e físico-química de barra de cereal de caju. Revista Brasileira de Tecnologia Agroindustrial, v. 7, n. 1, p. 934-942, 2013.

PINHO, L. X. Aproveitamento do resíduo do pendúnculo de caju (Anarcadium occidentale L.) para alimentação humana. 85 f. Dissertação (Mestrado em Ciência e Tecnologia de Alimentos) - Universidade Federal do Ceará, Fortaleza, 2009.

PEIXOTO, M.; PINTO, H. S. Desperdício de Alimentos: questões socioambientais, econômicas e regulatórias. Brasília: Núcleo de Estudos e Pesquisas/CONLEG/Senado, fevereiro/2016 (Boletim Legislativo no 41, de 2016). Disponível em:

<www.senado.leg.br/estudos>.Acesso em: 29 de nov. de 2016.

PORPINO, G. Comida: Brasil joga fora mais do que o necessário para combater a insegurança alimentar. Pagina 22, FGV/EAESP, São Paulo, 2 dez. 2016. Disponível em:< http://pagina22.com.br/2016/12/02/comida-brasil-joga-fora-mais-do-queo-necessario-para-combater-inseguranca-alimentar/>. Acesso em: 10 jan. 2017.

RUFINO, M. S. M.; PÉREZ-JIMÉNEZ, J.; TABERNERO, M.; ALVES, R. E.; BRITO, E. S.; SAURA-CALIXTO, F. Acerola and cashew apple as sources of antioxidants and dietary fibre. International Journal of Food Science and Technology, Endinburgh, v. 45, p. 2227-2233, 2010.

SABINO, V. G.; OLIVEIRA, E. N. A. de; FEITOSA, B. F.; FEITOSA, R. M.; OLIVEIRA, S. n, de. Desenvolvimento e caracterização de biscoitos tipo cookie de farinha do resíduo agroindustrial do caju. Revista Brasileira de Agrotecnologia, v. 7, n. 2, p. 38-44, 2017.

SILVA, A. F. O.; GIRONDI, L. M.; PIMENTEL, T. C.; BARÃO, C. E.; KLOSOSKI, S, J. Barra 
sensorial. Brazilian Journal of Food Research, Campo Mourão, v. 7, n. 2, p. 42-52, mai./ago. 2016.

SUCUPIRA, A, N. R.; SOUSA, P. H. M.; CONSTANT, P. B. L.; CACAU, M. S. C.; REBOUÇAS, J. Elaboração e aceitação sensorial de doce de coco substituído parcialmente por fibra de pedúnculo de caju. Revista Brasileira de Produtos Agroindustriais, Campina Grande, v. 16, n. 2, p. 213-216, 2014.

UCHOA, A. M. A.; COSTA, J. M.; MAIA, G. A.; SILVA, E. M. C.; CARVALHO, A. F. F. U.; MEIRA, T. R. Parâmetros Físico-Químicos, Teor de Fibra Bruta e Alimentar de Pós Alimentícios Obtidos de Resíduos de Frutas Tropicais. Segurança Alimentar e Nutricional, v. 15, n. 2, p. 58-65, 2008.

VIEIRA, C. F. S.; MARTINS, G. A. de S.; BORGES, S. V.; CARNEIRO, J. D. S.; REGES, I. S. Utilização de farinha de casca de maracujá amarelo em bolo. Enciclopédia Biosfera, v. 6, n. 11, p. 1-10, 2010.

MORAIS, E. C. et al. Elaboração de cupcake adicionado de farinha de fibra de caju: caracterização físicoquímica e sensorial. Brazilian Journal of Food Research, Campo Mourão, v. 9, n. 2, p. 1-14, abr./jun. 2018. Disponível em: https://periodicos.utfpr.edu.br/rebrapa

\section{Correspondência:}

Departamento de Ensino, Pesquisa e Extensão, Instituto Federal de Mato Grosso, Campus Cuiabá, Av. Juliano Costa Marques, s/n - CEP: 78.050-560, Cuiabá, Mato Grosso, Brasil.

Direito autoral: Este artigo está licenciado sob os termos da Licença Creative Commons-Atribuição 4.0 Internacional. 